\title{
Mini-review and our experience of methimazole- related omphalomesenteric duct remnant, omphalocele, and esophageal atresia
}

\begin{abstract}
Methimazole (MMI), carbimazole (CMZ), a prodrug of MMI, and propylthiouracil (PTU), are used to treat hyperthyroidism during pregnancy. However, the use of $\mathrm{MMI} / \mathrm{CMZ}$ in pregnancy, particularly during organogenesis, is subject to precautions or warnings because of the risk of neonatal congenital malformations. The risk of congenital malformations is recognized, but the epidemiology of MMI/CMZ-related congenital malformations is not well known in Japan. Our mini-review introduced the recent papers and our retrospective study, which evaluated the medical records of 68patients with surgery for omphalomesenteric duct remnant, omphalocele, and esophageal atresia between 1991 and 2010 and the treatment history of the mothers to determine use of MMI during pregnancy. Our study revealed the mothers of six of the 68patients had Graves' disease and all had been treated with MMI during pregnancy. No mother of any patient undergoing surgery for these conditions had been treated with MMI during pregnancy after the package insert had been revised to inform MMI prescription is contraindicated in early gestational weeks, leading to restricted prescription in Japan. Our data is consistent with the current notion of MMI/CMZ embrynopathy, the teratogenicity of these drugs. (187words).
\end{abstract}

Keywords: omphalomesenteric duct remnant, omphalocele, esophageal atresia, methimazole, pregnancy
Volume 3 Issue 4 - 2017

\author{
Yukihiro Hasegawa,' Hiroyuki Shinohara, ${ }^{1,2}$ \\ Seiichiro Hirobe ${ }^{3}$ \\ 'Division of Endocrinology and Metabolism, Tokyo Metropolitan \\ Children's Medical Center, Japan \\ ${ }^{2}$ Department of Pediatrics, University of Tsukuba, Japan \\ ${ }^{3}$ Division of Surgery, Tokyo Metropolitan Children's Medical \\ Center, Japan
}

\begin{abstract}
Correspondence: Yukihiro Hasegawa, Division of Endocrinology and Metabolism, Tokyo Metropolitan Children's Medical Center, 2-8-29 Musashi-dai, Fuchu, Tokyo, Japan, Email yhaset@gmail.com
\end{abstract}

Received: December 23, 2017 | Published: December 28, 2017
Abbreviations: MMI, methimazole; CMZ, carbimazole; PTU, propylthiouracil

\section{Introduction}

Because gestational hyperthyroidism is associated with maternal complications such as preeclampsia, premature labor, and premature delivery, ${ }^{1}$ hyperthyroidism must be treated during pregnancy. Methimazole (MMI) and carbimazole (CMZ), a prodrug of MMI, and propylthiouracil (PTU) are indicated for gestational hyperthyroidism, ${ }^{2}$ but $\mathrm{MMI} / \mathrm{CMZ}$ use during fetal organogenesis in the first trimester of pregnancy should be avoided ${ }^{3,4}$ because of the risk of neonatal congenital malformations. Some MMI/CMZ-related birth defects, such as omphalomesenteric duct remnant, omphalocele, and esophageal atresia require surgical treatment, ${ }^{3,4}$ whereas different, less severe defects are associated with PTU. ${ }^{5}$ Evidence provided by recent meta-analyses has resulted in labeling warnings and precautions on the use of MMI/CMZ, but not PTU, by pregnant women during fetal organogenesis. ${ }^{6,7}$ However, because these three conditions rarely occur in the general population, the MMI/CMZ-related incidence is not officially known in Japan. In this Mini-Review, we tried to show whether the above-mentioned congenital malformations needing surgical treatment at our institution were associated with maternal use of MMI during pregnancy by using our patients' cohort.

\section{What we did and found out}

The 76patients who underwent surgery for omphalomesenteric duct remnant, omphalocele, or esophageal atresia at our institution between 1991 and 2010 were identified from their medical records.
The records were retrospectively reviewed to determine whether the mothers had pregnancies complicated by Graves' disease and whether they had used oral MMI or PTU preceding pregnancy. The Institutional Review Board of TMCHC approved the study (H29-28).

Eight of the 76 subjects were excluded for chromosomal abnormalities such as Down syndrome; the remaining 68 were included in the evaluation. Twelve of the 68 congenital abnormalities were omphalomesenteric duct remnants, 14 were omphaloceles, and 46esophageal atresias. Four patients had both omphalomesenteric duct remnants and omphaloceles, and one patient had an omphalomesenteric duct remnant complicated by scalp defects, which is also a characteristic MMI/CMZ-related birth defect. Six of the mothers had Graves' disease, all had been treated with MMI during pregnancy, and none received other hyperthyroid medications such as PTU or potassium iodide. All six of these pregnancies occurred before the year 2000(six of 51 before 2000), whereas no mothers (7 in total after 2000) had Graves' disease or used MMI during their pregnancy. The use of MMI and the characteristics of the cases with the three congenital abnormalities are shown in Table 1. The mothers of five of 12 infants with omphalomesenteric duct remnant, two of 14 with omphalocele, and one of 46 with esophageal atresia had used MMI. The anomaly most frequently associated with MMI (42\%) was omphalomesenteric duct remnant followed by omphalocele $(14 \%)$, and both percentages were significantly higher than that of esophageal atresia $(2 \%, p<0.01)$. The difference indicates that omphalomesenteric duct remnant and omphalocele, although fewer in number for patients than esophageal atresia, may be more closely associated with MMI use during pregnancy. 
Table I Omphalomesenteric duct remnant, omphalocele, and esophageal atresia with or without maternal MMI use during pregnancy

\begin{tabular}{llllll}
\hline MMITx & ODR Alone & ODR+OP & OP Alone & EA & Total \\
\hline$(+)$ & 3 & 2 & 0 & 1 & 6 \\
$(-)$ & 5 & 2 & 10 & 45 & 62 \\
Total & 8 & 4 & 10 & 46 & 68 \\
\hline
\end{tabular}

ODR, omphalomesenteric duct remnant; OP, omphalocele; EA, esophageal atresia

The proportions of ODR (5/12) and OP (2/14) were greater than that of EA $(\mathrm{I} / 46), \mathrm{p}<0.0 \mathrm{I}$

\section{What we would like to discuss in this study}

In this study, all of the mothers with gestational Graves' disease and children with surgery for neonatal omphalomesenteric duct remnant, omphalocele, or esophageal atresia had been treated with MMI. This result is consistent with that reported by a recent metaanalyses.${ }^{6,7}$ None of the conditions evaluated and treated after the year 2000 occurred in children with mothers who had been treated with MMI during pregnancy. This can be attributed to the fourth revision of the MMI package insert in 1999, which restricted prescription of MMI during the early phase of pregnancy in Japan. Unfortunately, no official national or district data on the incidence of these three congenital abnormalities are available in Japan. It was thus not possible to compare the study results with a recent estimate of incidence in the general population.

To the best of our knowledge, this is the first study to investigate the association of these three rare surgically treated conditions and maternal MMI use during the preceding pregnancy. This kind of study would be difficult to perform anywhere other than a specialized pediatric hospital like this one, because of a lack of eligible cases. The evidence from 11 clinical studies that confirmed the teratogenicity has been recently reviewed. ${ }^{7}$ One of those studies, conducted in Japan by Yoshihara et al. and published in $2012^{4}$ evaluated the same surgically treated MMI-associated surgical conditions in nearly 7000 pregnancies complicated by Graves' disease. The study provided evidence of the teratogenicity of MMI because the incidence of birth defects in the MMI group was higher than in a control group receiving no Graves' disease treatment. However, no cases requiring intervention were observed at birth in the control group, making statistical comparison and its interpretation between the MMI and control group difficult. Many more participants would have been needed to do the appropriate statistical analysis. Additional evidence of the teratogenicity of MMI has been provided by an interim report of the POEM (Pregnancy Outcomes of Exposure to Methimazole) study that began in $2008^{8}$ and a nationwide retrospective study conducted in children born in Denmark between 1996 and 2008. ${ }^{9}$

Our study had at least three limitations. The first was that the mothers' medical history lacked detailed MMI treatment information and the results of thyroid tests. It is unlikely that this significantly influenced the results because the most important factor determining the teratogenicity of MMI is use in the first trimester of pregnancy. The actual dose is known not to be relevant, ${ }^{5}$ and the results of maternal thyroid function tests have not been found to influence the incidence of birth defects. ${ }^{1}$ Secondly, the medical records may have lacked some maternal history related to Graves' disease and MMI, which might have resulted in underestimating the association of these rare surgically treated conditions and gestational use of MMI. The third limitation was the retrospective design and small number of subjects, which was too small to draw a definitive conclusion, included at only one institution. A prospective, multicenter study would provide more accurate information about the mothers, but would also require a much longer amount of time to collect the needed information about the occurrence of these rare conditions. In addition, because prescription of MMI is not recommended during the early phase of pregnancy (5), any prospective study including MMI administration during pregnancy would pose ethical problems.

Despite its limitations, the study is unique in using patient cohorts with the three surgical conditions, and allows a practical option to infer the relationship between MMI and its rare complications. This study strategy may be useful in making interferences about the teratogenicity of drugs and their association with rare birth defects. This evaluation confirms that MMI-related embryopathy of omphalomesenteric duct remnant, omphalocele, and esophageal atresia disappeared at our institution after the revision of the MMI package insert in 1999.

\section{Conclusion}

Our data is consistent with the current notion of $\mathrm{MMI} / \mathrm{CMZ}$ embryopathy, the teratogenicity of these drugs.

\section{Acknowledgements}

YH and HS contributed equally to this work

\section{Conflict of interest}

Author declares that there is no conflict of interest.

\section{References}

1. Mannisto T, Mendola P, Grewal J, et al. (2013) Thyroid diseases and adverse pregnancy outcomes in a contemporary US cohort. J Clin Endocrinol Metab. 2013;98(7):2725-2733.

2. Prunty JJ, Heise CD, Chaffin DG. Graves' disease pharmacotherapy in women of Reproductive age. Pharmacotherapy. 2016;36(1):64-83.

3. Momotani N, Ito K, Hamada N, et al. Maternal hyperthyroidism and congenital malformation in the offspring. Clin Endocrinol (Oxf). 1984;20(6):695-700.

4. Yoshihara A, Noh J, Yamaguchi T, et al. Treatment of graves' disease with antithyroid drugs in the first trimester of pregnancy and the prevalence of congenital malformation. J Clin Endocrinol Metab. 2012;97(7):2396-2403.

5. Andersen SL. Risk of embryopathies with use of antithyroidal medications. Curr Opin Endocrinol Diabetes Obes. 2017;24(5):364-371.

6. Li X, Liu GY, Ma JL, et al. Risk of congenital anomalies associated with antithyroid treatment during pregnancy: a meta-analysis. Clinics (Sao Paulo). 2015;70(6):453-459.

7. Song R, Lin H, Chen Y, et al. Effects of methimazole and propylthiouracil exposure during pregnancy on the risk of neonatal congenital malformations: A meta-analysis. PLoS One. 2017;12(7):e0180108.

8. Arata N. Pregnancy outcomes of exposure to methimazole (POEM) study. Nihon Rinsho. 2012;70(11):1976-1982.

9. Andersen SL, Olsen J, Wu CS, et al. Birth defects after early pregnancy use of antithyroid drugs: a Danish nationwide study. J Clin Endocrinol Metab. 2013;98(11):4373-4381. 